\title{
Cizallamiento para uniones con clavos de caña lisa y helicoidal en maderas de Bombacaceae
}

\author{
Shear for nail joints with plain-shank and helically nails on Bombacaceae wood
}

\author{
Mariano Vila Meneses ${ }^{1}$ y Miguel Meléndez Cárdenas ${ }^{2}$
}

\begin{abstract}
Resumen
El presente trabajo trata sobre la determinación de cargas admisibles para uniones con clavos de caña lisa y helicoidal, sometidas a simple y doble cizallamiento en madera de Ceiba samauma (huimba), Matisia bicolor (machin zapote) y Matisia cordata (zapote); observándose una ventajosa diferencia por el uso del clavo de caña helicoidal. Los ensayos y cálculos respectivos se efectuaron de acuerdo a las consideraciones de la Norma ASTM D-1761-74 y la Sección de Construcciones Forestales del Departamento de Industrias Forestales. Asimismo se discute el comportamiento de las uniones estudiadas, analizando sus cargas máximas a través del experimento factorial 3A2B2C, bajo un diseño completamente randomizado.
\end{abstract}

Palabras clave: uniones clavadas; cizallamiento; Bombacaceae; clavo caña lisa; clavo helicoidal.

\begin{abstract}
The present work is about the determination of the allowable loads for joints of plainshank and helically nails that were evaluated in simple and double shearing for woods Ceiba samauma (huimba), Matisia bicolor (machin zapote) and Matisia cordata (zapote); the results indicated that the best perfomance was the helically nail. The study and calculations were made in accord with the considerations of the ASTM D-1761-74 Norm and the Forestry Construction Section of Forestry Industry Departament. Moreover, the joints behaviour are discussed, making an analysis of their maximum loads by the factorial experiment 3A2B2C in a completely random design.
\end{abstract}

Key words: nail joints; shearing; Bombacaceae; plain-shack nail; helically nail.

\footnotetext{
${ }^{1}$ Consultor independiente, Perú.

${ }^{2}$ Facultad de Ciencias Forestales, Universidad Nacional Agraria La Molina, Perú. E-mail: mmecd@lamolina.edu.pe
} 


\section{Introducción}

Cuando se construye con madera se tiene que asumir el reto de solucionar bien las uniones, si se desea conferir calidad, durabilidad y rentabilidad a la construcción con este material. En el diseño estructural la concepción de tales uniones está enteramente ligada al elemento de ensamble utilizado.

El estudio de las uniones estructurales constituye un aspecto prioritario en el diseño con madera. Así tenemos que una unión débil puede convertir una estructura en ineficaz o insegura, y que el comportamiento de ésta será inadecuado si las uniones no permiten desarrollar su capacidad resistente a los elementos unidos.

Robles y Echenique (1989) sostienen que las uniones clavadas más eficaces son las que aprovechan la capacidad para transmitir fuerza lateral, y que este tipo de uniones son sencillas y económicas, siendo usadas con preferencia en estructuras ligeras en las que el grosor de las piezas por unir es inferior a unos cinco centímetros.

Stern y Stoneburner citado por Takahashi (1967) indican que ensayos hechos con juntas de madera seca, resisten mayores cargas que las confeccionadas con maderas verdes, pero aumenta la tendencia al rajado.

Centeno (1976) señala que la capacidad estructural de una unión clavada o empernada depende principalmente de: las propiedades del elemento de unión, clavo o perno, las propiedades de la madera, la interacción creada por la combinación y distribución de los factores anteriores y el tipo de esfuerzo aplicado.

Scholten, citado por Campos y Cano (1996), indica que la resistencia, estabilidad y vida útil de una estructura o parte de ella depende de la magnitud de la resistencia, rigidez y durabilidad de las juntas. Explica a su vez la influencia de la madera, el clavo y las condiciones de uso en la eficiencia de las uniones clavadas. Respecto a la madera enumera los siguientes factores: peso específico, contenido de humedad y dirección del clavado respecto a la fibra.
Los autores antes citados, al igual que Hoyle (1978), Breyer (1980) y Robles y Echenique (1989) coinciden con las siguientes consideraciones de la National Buildings Organization sobre esfuerzos y economía de las juntas clavadas: tipo de junta, especie de madera, diámetros de los clavos, número de clavos, distribución de clavos en la unión, penetración de los clavos en los elementos principales y secundarios, esfuerzos de los clavos y método de hincado.

La sección ocho de la National Design Specification (NDS), consultada por Campos y Cano (1996), hace referencia al efecto de la punta del clavo en la penetración del mismo y el esfuerzo de la junta, por ello recomienda un pre-taladrado, que para el caso de maderas duras no deberá exceder de los $4 / 5$ del diámetro del clavo y para maderas suaves los $3 / 5$. Cuando un agujero pretaladrado tiene un diámetro que no excede los $4 / 5$, de los clavos usados, la carga permisible aplicada en la resistencia lateral y de extracción es la de un clavo sin agujero pre-taladrado.

Breyer (1980) agrega otros factores de importancia como: dirección del clavado longitudinal y transversal, contenido de humedad en la instalación y en uso y duración de la carga.

Takahashi (1967), Robles y Echenique (1989) coinciden en resaltar las ventajas del uso de clavos con estrías helicoidales, pues son especialmente útiles cuando la madera está verde, ya que conservan considerable resistencia a la extracción aún después de la contracción que sufre la madera al secarse. Stern (1960) detalla las siguientes ventajas para éstos clavos:

Las cargas de diseño transmitidas por varios clavos comunes pueden ser reemplazados por un solo clavo helicoidal.

Se puede aprovechar mejor la madera de menor calidad. Concluye que el aumento en el costo por el uso de estos clavos es casi despreciable desde el punto de vista del costo total, dada las grandes ventajas debido a su uso.

Scholten, citado por Campos y Cano (1996), menciona en cuanto al clavo de caña helicoidal, 
que mejora la seguridad en la junta, la cual a diferencia de los de caña lisa, no está sujeta a reducciones de carga. Por último, este tipo de clavos tienden a producir menores rajaduras, debido a sus diámetros menores en relación a sus longitudes que los clavos comunes.

La unión entre elementos de madera dispone de diferentes alternativas de fijación, entre las cuales, los clavos figuran como el medio más fácil y conveniente. Tradicionalmente en nuestro medio se preparan uniones con clavos de caña lisa, no obstante el clavo de caña helicoidal representa una nueva opción para las uniones en maderas corrientes.

Considerando la abundancia de especies secundarias con buenas características técnicas pero limitadas aplicaciones, resulta conveniente promover a maderas como las Bombacaceae, las cuales representan una oportunidad de vanguardia dentro de la actual política mundial de aprovechamiento de maderas tropicales.

El presente trabajo tiene por objetivos determinar las cargas admisibles para uniones con clavos de caña lisa y helicoidal, sometidas a simple y doble cizallamiento; así como diferenciar el comportamiento de ambos tipos de clavos en dichas uniones.

\section{Materiales y Métodos}

\section{Lugar de ejecución}

El estudio se realizó en el Laboratorio de Ensayos Tecnológicos del Departamento Académico de Industrias Forestales y en el Centro de Estadística y Procesamiento de Datos, de la Universidad Nacional Agraria La Molina (Lima, Perú), en el año 1994.

\section{Materiales y equipo}

a) Madera; se prepararon probetas de madera recolectada en la Unidad de Manejo y Producción Forestal Dantas, y proveniente de tres especies forestales: Ceiba samauma (Mart.) Schum. (huimba negra), Matisia bicolor Ducke. (machin zapote) y Matisia cordata Humb. et Bonpl. (zapote). b) Clavos; se emplearon clavos de alambrón trefilado con cabeza y punta en forma de diamante, de 3" de longitud, calibre 9, y dos tipos de caña: lisa y helicoidal.

\section{c) Equipo:}

- Máquina universal de ensayos Tinus Olsen, con sus accesorios

- Deflectómetros con una carrera de 25 mm y una precisión de $0,01 \mathrm{~mm}$.

- Cronómetro

- Vernier digital

- Microcomputadora 386-SX con los programas: Word Perfect 5.1, Lotus 123 R 2.4 y Harvard Graphic 3.0.

\section{- Impresora Epson LX-810}

\section{Metodología}

\section{Diseño del experimento}

Las probetas ensayadas se diseñaron para uniones con cuatro clavos hincados de un solo lado, a simple y doble cizallamiento, tomando en cuenta las consideraciones de la JUNAC (1980). Para cada especie se ensayaron 40 probetas distribuidas como sigue:

1. Ensayo de uniones clavadas a simple cizallamiento:

- Con clavos de caña lisa: 10

- Con clavos de caña helicoidal: 10

2. Ensayo de uniones clavadas a doble cizallamiento:

- Con clavos de caña lisa: 10

- Con clavos de caña helicoidal: 10

\section{Elaboración de las probetas}

Utilizando distintas máquinas de carpintería se prepararon piezas de madera libre de defectos, cepillada y orientada arbitrariamente. Para evitar posibles rajaduras en la madera y asegurar el hincamiento recto del clavo se pretaladraron las probetas, con un agujero del orden de 0,8 veces el diámetro del clavo, realizado con una broca extralarga de 1/8" de diámetro.

En ambos tipos de cizallamiento se consideró una relación $\mathrm{L} / \mathrm{D}$ de las probetas de 10,6 
para clavos de caña lisa y 10,9 para clavos de caña helicoidal.

\section{Ensayo de las uniones clavadas}

Se siguieron los procedimientos establecidos por la Norma ASTM D-1761-74 con algunas modificaciones. Concluido el ensayo, se obtuvieron muestras de $2 \mathrm{~cm}$ de longitud de cada elemento, para la determinación del contenido de humedad de las probetas, según la Norma Técnica ITINTEC 251.010.

Con los datos obtenidos se graficó la curva carga-deformación y se calculó la carga correspondiente a: la deformación $0,038 \mathrm{~cm}$ $\left(0,015^{\prime}\right)$, el límite proporcional y la deformación máxima.

\section{Procesamiento de datos}

Las lecturas registradas en los formatos de prueba respectivos, se introdujeron en la hoja electrónica de cálculo Lotus 123, para el procesamiento automatizado de la información requerida en los diagramas carga-deformación.

\section{Análisis estadístico}

La carga máxima originada por dos tipos de clavos sometidos a simple y doble cizallamiento en uniones con tres especies forestales, se analizó estadísticamente a través de un diseño completamente randomizado con arreglo factorial 3A2B2C detallado como sigue a continuación:

Factor A: especie.

Factor B: tipo de cizallamiento.

Factor C: tipo de caña del clavo.

Niveles del Factor Especie: huimba, machin zapote y zapote.

Niveles del Factor tipo de cizallamiento: simple y doble.

Niveles del Factor tipo de caña del clavo: lisa y helicoidal.

Material experimental: madera aserrada de las especies huimba, machin zapote y zapote.

Unidad experimental: probeta.

Variable observada: carga máxima en kg.

\section{Elaboración del diagrama carga-defor- mación de los ensayos}

Los diagramas Carga-Deformación se confeccionaron utilizando el programa de diseño gráfico Harvard Graphic 3.0, para lo cual se importaron los datos introducidos a la hoja de cálculo Lotus 123, graficando en el eje de las ordenadas las cargas en kilos y en el eje de las abcisas la deformación en centímetros.

\section{Elaboración de las tablas de diseño}

Los valores de diseño, fueron calculados bajo las pautas dadas en la Sección de Construcciones Forestales de la UNALM (Cano y Sato 1968).

La carga admisible por clavo $\left(\mathrm{P}_{\text {adm }} /\right.$ clavo $)$ se obtuvo de la siguiente manera:

$$
\mathrm{P}_{\mathrm{adm}}=\underline{\mathrm{P}}_{\underline{\max }} \frac{-\mathrm{t}_{0,05}}{3} \underline{\mathrm{xS}}
$$

Donde:

$\mathrm{P}_{\text {adm }} /$ clavo $=\mathrm{P}_{\text {adm }} / 4$

$\mathrm{P}_{\max }=$ promedio de las cargas máximas.

$\mathrm{t}_{0,05}=$ valor de la tabla " $\mathrm{t}$ " para $\mathrm{n}-1 \mathrm{gl}$ y $95 \%$ de probabilidad.

$\mathrm{n}=$ número de repeticiones.

S=desviación estándar total.

\section{Resultados}

\section{Carga lateral en uniones clavadas}

En los Cuadros 1, 2 y 3 se aprecia respectivamente para cada especie, la carga lateral promedio por cada tipo de unión clavada. Estos cuadros permiten distinguir el siguiente comportamiento:

En el límite proporcional; las uniones a simple cizallamiento exhiben una marcada tendencia hacia mayores valores en aquellas confeccionadas con clavos de caña lisa. Sin embargo en las uniones a doble cizallamiento no se manifiesta la misma tendencia.

En la deformación $0,038 \mathrm{~cm}$; los mayores valores de carga corresponden a las uniones con clavos de caña lisa, a simple y doble cizal- 


\begin{tabular}{|c|c|c|c|c|}
\hline Tipo de cizallamiento & \multicolumn{2}{|r|}{ Simple } & \multicolumn{2}{|r|}{ Doble } \\
\hline Tipo de caña & Lisa & Helic. Var\% & Lisa & Helic. Var\% \\
\hline 1. $\mathrm{P}_{\text {máx }}$ prom. $(\mathrm{Kg})$ & 708,2 & $1258,577,7$ & 12530 & $1778,3 \quad 41,9$ \\
\hline CV $(\%)$ & 18,6 & 14,7 & 17,0 & 12,9 \\
\hline 2. $\mathrm{P}_{\mathrm{ELP}}$ prom. $(\mathrm{Kg})$ & 270,7 & 176,0 & 206,3 & 258,7 \\
\hline CV $(\%)$ & 35,5 & 18,3 & 22,6 & 32,0 \\
\hline 3. $\mathrm{P}_{0,038}$ prom. $(\mathrm{Kg})$ & 390,9 & 269,1 & 447,6 & 408,4 \\
\hline CV $(\%)$ & 27,1 & 25,5 & 25,2 & 24,9 \\
\hline
\end{tabular}

Cuadro 1. Carga lateral promedio para tres niveles de deformación en uniones clavadas con huimba.

\begin{tabular}{|c|cc|cc|}
\hline Tipo de cizallamiento & \multicolumn{2}{|c|}{ Simple } & \multicolumn{2}{c|}{ Doble } \\
\hline Tipo de caña & Lisa & Helic. Var\% & Lisa & Helic. Var\% \\
\hline 1. P max $_{\text {mrom. }}(\mathrm{Kg})$ & 992,9 & $1488,749,9$ & 1441,9 & $1802,425,0$ \\
CV (\%) & 14,7 & 10,9 & 8,7 & 6,0 \\
\hline 2. PLLP $_{\text {prom. }}(\mathrm{Kg})$ & 230,9 & 201,2 & 270,1 & 265,8 \\
CV (\%) & 30,1 & 22,2 & 20,5 & 18,3 \\
\hline 3. P $_{0,038}$ prom. $(\mathrm{Kg})$ & 393,1 & 360,4 & 523,5 & 483,1 \\
CV (\%) & 31,0 & 12,9 & 21,4 & 19,8 \\
\hline
\end{tabular}

Cuadro 2. Carga lateral promedio para tres niveles de deformación en uniones clavadas con machin zapote.

\begin{tabular}{|c|cc|cc|}
\hline Tipo de cizallamiento & \multicolumn{2}{|c|}{ Simple } & \multicolumn{2}{c|}{ Doble } \\
\hline Tipo de caña & Lisa & Helic. Var\% & Lisa & Helic. Var\% \\
\hline 1. P $_{\text {móx }}$ prom. $(\mathrm{Kg})$ & 856,9 & $1232,643,8$ & 1043,7 & $1459,639,8$ \\
CV (\%) & 28,9 & 26,5 & 26,6 & 25,0 \\
\hline 2. P $_{\text {ELP }}$ prom. $(\mathrm{Kg})$ & 226,4 & 171,8 & 191,4 & 256,4 \\
CV (\%) & 36,8 & 18,8 & 32,4 & 32,1 \\
\hline 3. P $_{0,038}$ prom. $(\mathrm{Kg})$ & 343,9 & 226,8 & 342,1 & 334,6 \\
CV (\%) & 39,6 & 30,1 & 36,0 & 44,3 \\
\hline
\end{tabular}

Cuadro 3. Carga lateral promedio para tres niveles de deformación en uniones clavadas con zapote.

lamiento, lo cual se traduce en una mayor pendiente de la línea elástica de los diagramas construídos.

En la carga máxima; los mayores valores, tanto en el simple como en el doble cizallamiento, corresponden a las uniones con clavos de caña helicoidal.

\section{Análisis de variancia de la carga máxima}

La alta significancia del análisis inicial de variancia de la carga máxima obtenida del experimento factorial, muestra indicios de la influencia de los factores considerados como principales: especie, tipo de cizallamiento y tipo de caña. La influencia de estos factores se visualiza discriminadamente en el Cuadro 4, en el que además sobresale el efecto conjunto de la especie con el tipo de cizallamiento. Vila (1995) complementa el análisis de los efectos principales con las pruebas de DLS, Duncan y Tukey, y el análisis de efectos simples para la interacción especie-tipo de cizallamiento.

Por las significancias observadas a nivel de carga máxima, se ha podido confirmar algunas consideraciones reportadas en la revisión de literatura (especie, tipo de cizallamiento y tipo de caña), así como encontrar la interacción de los factores especie-tipo de cizallamiento. De los tres factores analizados el efecto del tipo de caña en las uniones clavadas, merece una consideración especial por la bondad comprobada debido al uso del clavo helicoidal, lo cual jus- 

en maderas de Bombacaceae

tifica un factor de ajuste por tipo de caña en el cálculo de las cargas admisibles a partir de sus cargas máximas.

\section{Cargas admisibles por clavo}

En el Cuadro 5 se proporcionan cargas admisibles de acuerdo al tipo de cizallamiento y clavo, para uniones con madera seca al $12 \%$ y clavos de 3" de longitud. Estas cargas se consideran conservadoras, pues ninguna de ellas superarían la carga correspondiente a la deformación 0,038 cm, mostrada en los Cuadros 1 , 2 y 3 , con lo cual se tiene un margen de holgura en el diseño respectivo. De tal forma que si una estructura alcanzara dicha deformación, estaríamos completamente seguros con la carga admisible utilizada.

\section{Discusión}

La tendencia observada en la carga lateral en uniones clavadas se refleja en los diagramas carga-deformación graficados por Vila (1995). En cuanto a las uniones con clavos de caña lisa y helicoidal, los resultados revelan un marcado comportamiento en dos aspectos:

a) Mayor resistencia en las uniones con clavos de caña helicoidal

La ganancia porcentual en carga máxima promedio, como resultado del cambio de clavos lisos por helicoidales, conlleva a pensar que la bondad del clavo helicoidal como medio de unión de elementos de madera, radica en su capacidad para soportar altas cargas de trabajo debido a la característica enhebrada de su caña. La introducción en la madera de este tipo de caña, probablemente ocasiona un acomodo de las fibras entorno a dicha caña, confiriéndole una mayor superficie de contacto y de fricción.

\begin{tabular}{|c|cr|cc|}
\hline \multirow{2}{*}{ Especie } & \multicolumn{3}{|c|}{ Carga admisible por clavo en kg (n) } \\
\cline { 2 - 5 } & \multicolumn{2}{|c|}{$\begin{array}{c}\text { Cizallamiento } \\
\text { simple }\end{array}$} & \multicolumn{2}{c|}{$\begin{array}{c}\text { Cizallamiento } \\
\text { doble }\end{array}$} \\
\cline { 2 - 5 } & \multicolumn{2}{|c}{ C. Lisa C. Helic } & C. Lisa & C.Helic \\
\hline \multirow{2}{*}{ Huimba } & 34 & 69 & 64 & 104 \\
& $(333)$ & $(677)$ & $(628)$ & $(1020)$ \\
\hline Machin & 55 & 93 & 96 & 129 \\
zapote & $(539)$ & $(912)$ & $(941)$ & $(1265)$ \\
\hline \multirow{2}{*}{ Zapote } & 24 & 40 & 33 & 51 \\
& $(235)$ & $(392)$ & $(324)$ & $(500)$ \\
\hline
\end{tabular}

Cuadro 5. Cargas admisibles por clavo para tres especies forestales en condición seca al $12 \%$ y con clavos de 3 ".

Esta mayor capacidad permite reducir la densidad de clavos, la cual posiblemente abarate de algún modo el mayor costo de éste; aunque expertos como Stern (1960) señalan, que estos costos resultan al final despreciables, dada las múltiples ventajas de su empleo.

b) Menor rigidez en las uniones con clavos de caña helicoidal

La menor rigidez registrada en la unión con el clavo de caña helicoidal, podría explicarse en parte por la mayor esbeltez del clavo en la unión, debida a su menor sección, como producto de la peculiar geometría de estos clavos. Además de una menor presión transmitida sobre las paredes del agujero en la madera.

El análisis estadístico de los efectos principales, asigna al clavo de caña helicoidal una alta resistencia lateral y por ende, corrobora la afirmación de Stern (1960) referente a la utilización de un menor número de clavos en una unión fabricada con este tipo de caña. De ahí que el efecto producido por un determinado número de clavos de caña lisa puede ser

\begin{tabular}{|c|c|c|c|c|c|}
\hline Fuente & GL & SC & $\begin{array}{c}\text { Cuadrado } \\
\text { Medio }\end{array}$ & Valor F & Pr>F \\
\hline Especie & 2 & 1647863,91 & 823931,99 & 16,37 & 0,0001 \\
\hline Cizall. & 1 & 4185676,33 & 4185676,33 & 83,19 & 0,0001 \\
\hline Especie-Cizall. & 2 & 530373,76 & 265186,88 & 5,27 & 0,0065 \\
\hline Caña & 1 & 6180424,96 & 6180424,96 & 122,83 & 0,0001 \\
\hline Especie-Caña & 2 & 110756,16 & 55378,08 & 1,10 & 0,3364 \\
\hline Cizall-Caña & 1 & 12015,01 & 12015,01 & 0,24 & 0,6261 \\
\hline Especie-Cizall-Caña & 2 & 39341,05 & 19670,52 & 0,39 & 0,6774 \\
\hline
\end{tabular}

Cuadro 4. Análisis de los efectos principales en la carga máxima para tres especies forestales. 
reemplazado por un menor número de clavos de caña helicoidal. Este hecho favorece el uso de especies desconocidas en la construcción, como la huimba y el machin zapote, con las cuales se puede rigidizar una estructura utilizando tan sólo un tercio de los clavos requeridos para una unión con clavos comunes.

En términos generales se aprecia un incremento del valor de la carga admisible en las uniones con clavos de caña helicoidal. Este aumento es del orden de 1,8 y 1,5 veces la carga en uniones con clavos de caña lisa, para el simple y doble cizallamiento respectivamente.

Asimismo se deduce que las cargas en doble cizallamiento equivalen aproximadamente a 1,7 y 1,4 veces las cargas registradas en simple cizallamiento, para los clavos de caña lisa y helicoidal respectivamente.

También cabe destacar que, tanto en la carga máxima como en la carga admisible, los mayores valores corresponden a la especie machin zapote, seguida en orden decreciente por la huimba y el zapote.

\section{Conclusiones}

Los mayores valores de carga máxima y admisible en las uniones estudiadas corresponden a la especie machin zapote, seguida en orden decreciente por la huimba y el zapote.

Las cargas admisibles con clavos de caña lisa a doble cizallamiento superan en 1,7 veces las cargas a simple cizallamiento; del mismo modo con el empleo de clavos de caña helicoidal, este incremento es del orden de 1,4 veces las cargas a simple cizallamiento.

De acuerdo a las condiciones de la presente investigación, las cargas admisibles propuestas, revelan la excelente capacidad resistente de los clavos de caña helicoidal en las uniones estudiadas.

En un ensamble con madera, el número de clavos disminuye al cambiar de caña lisa a helicoidal, con la consiguiente reducción del riesgo de fisuración en la madera.

\section{Bibliografía}

Breyer, D. 1980. Design of wood structures. Mc Graw-Hill Book Co, New York. 542 p.

Cano, DJ; Sato, AA. 1968. Uniones clavadas de dos especies de Podocarpus. UNALM. Lima. $12 \mathrm{p}$.

Campos, MG; Cano, DJ. 1966. Uniones estructurales de madera Humiriastrum excelsum. Tesis Ingeniero Civil. Universidad Nacional de Ingeniería. Lima. 168 p.

Centeno, JC. 1976. Características mecánicas de uniones clavadas y empernadas de 20 especies de la Guayana Venezolana. Mérida-Venezuela. 23 p.

Hoyle, R. 1978. Wood technology in the design of structures. Mountain Press Publishing Co. Missoula, Montana. 390 p.

JUNAC. 1980. Normas y métodos para ensayos tecnológicos. Sub-proyecto $n^{\circ} 1$. Estudio de la tecnología e ingeniería de la madera. JUNAC. Bogotá-Colombia. 185 p.

Robles, FF; Echenique, MR. 1989. Estructuras de madera. México. 367 p.

Stern, GE. 1960. Wire nails. Virginia Polytechnic Institute. Wire. Blacksburg-Virginia. USA. 1004-1006 p.

Takahashi, SL. 1967. Estudio comparativo de uniones clavadas y encoladas-clavadas de quinilla colorada, sometidas a cizallamiento. Tesis Ingeniero Forestal. UNA La Molina. Lima. 100 p.

Vila, MM. 1995. Cizallamiento de uniones clavadas pretaladradas con clavos de caña lisa $y$ helicoidal en maderas de Bombacaceae. Tesis Ingeniero Forestal. UNA La Molina. Lima. 120 p. 